\title{
Plantas medicinais utilizadas na comunidade caiçara da Praia do Sono, Paraty, Rio de Janeiro, Brasil ${ }^{1}$
}

\author{
Mariana Reis de Brito ${ }^{2,3}$ e Luci de Senna-Valle ${ }^{2}$
}

Recebido em 17/09/2010. Aceito em 4/04/2011

\begin{abstract}
RESUMO
(Plantas medicinais utilizadas na comunidade caiçara da Praia do Sono, Paraty, Rio de Janeiro, Brasil). A comunidade caiçara da Praia do Sono ainda sustenta hábitos e tradições de antigas gerações, o que foi facilitado pelo difícil acesso a esta praia. O presente trabalho objetiva investigar o conhecimento sobre as plantas medicinais nesta comunidade. Foram realizadas entrevistas com 12 informantes especialistas ( 6 homens e 6 mulheres). As 89 espécies citadas estão distribuídas em 43 famílias, sendo Lamiaceae e Asteraceae as mais representativas. As indicações terapêuticas das plantas medicinais citadas foram agrupadas em 13 categorias de doenças, seguindo a CID-10, adotada pela OMS. As informações sobre as partes das plantas utilizadas, as indicações de uso e os locais de coleta foram indicados. Dez espécies apresentaram grande versatilidade quanto aos seus usos, com IR $>1$. Treze espécies, sendo Cecropia pachystachya Trec.; Davilla rugosa Poiret e Trema micrantha (L.) Blume nativas do bioma Mata Atlântica, apresentaram valores de CUPc maior que 50\%. Estes resultados servem de estímulo para novos trabalhos nessa área e para futuros estudos sobre os constituintes químicos e as propriedades farmacológicas destas plantas.
\end{abstract}

Palavras-chave: etnobotânica, comunidades litorâneas, abordagem quantitativa

\begin{abstract}
(Medicinal plants used in the "caiçara" community of Praia do Sono, Paraty, Rio de Janeiro, Brazil). The "caiçara" community of Praia do Sono retains some of the habits and traditions from ancient generations. This may be due to the difficulties of accessibility to that area. This paper aims to investigate the knowledge about the medicinal plants in this community. Twelve specialist informants were selected and interviewed (six men and six women). Eighty-nine species were cited, belonging to 43 botanical families. Lamiaceae and Asteraceae are the most representative ones. Therapeutic indications were decoded and grouped in 13 categories of diseases. This classification follows the ICD10 adopted by WHO. Information regarding the plant tissue used, their uses and the collection sites were indicated. Ten species showed great versatility of uses, with IR $>1$. Of the 13 species that have CUPc values over $50 \%$, Cecropia pachystachya Trec.; Davilla rugosa Poiret and Trema micrantha (L.) Blume are native to the Atlantic Forest. These results can be used as a motivation for further studies in the area and also can provide the basis for future studies on chemical constituents and pharmacological proprieties of the plants cited.
\end{abstract}

Key words: ethnobotany, coastal communities, quantitative approach

\section{Introdução}

Estudos etnobotânicos de registro de plantas, seus usos e formas terapêuticas por grupos humanos têm contribuído tanto para planos de conservação e manejo de ecossistemas (Prance 1995) como no campo da fitoquímica e farmacologia, inclusive como ferramenta para o descobrimento de novas drogas (Elisabetsky \& Souza 2004). Atualmente existe uma crescente produção de artigos científicos nesta área, refletindo uma conscientização do potencial do estudo etnobotânico, especialmente nas pesquisas de plantas medi- cinais. De acordo com a opinião endossada por Manzali de Sá (2006), o conhecimento de populações tradicionais sobre plantas biologicamente ativas é um elemento valorizado pelos pesquisadores que atuam na área de desenvolvimento de novos fármacos.

Seguindo a tradição, as plantas medicinais continuam sendo bastante empregadas na medicina popular de hoje em dia, no preparo de remédios caseiros. Este fato devese provavelmente às dificuldades impostas às populações pelos sistemas de saúde vigentes. As comunidades rurais dificilmente conseguem atendimento médico, devido a falta

\footnotetext{
Parte da dissertação de Mestrado da primeira Autora

2 Universidade Federal do Rio de Janeiro, Museu Nacional, Departamento de Botânica, Rio de Janeiro, RJ, Brasil

3 Autor para correspondência: marianareis2002@hotmail.com
} 
de uma unidade de saúde minimamente equipada e às más condições das estradas e transportes públicos.

A Mata Atlântica possui influência em todos os campos da cultura brasileira e, mais que qualquer bioma do país, guarda os marcos de nossa história. Portanto, sua destruição ameaça além da riqueza e diversidade florística, um rico patrimônio histórico e diversas comunidades tradicionais, que constituem parte importante da diversidade cultural do Brasil (MMA 2002). Assim, o estudo sobre os recursos medicinais em comunidades tradicionais está se tornando uma necessidade urgente, já que se corre o risco de perder não somente as espécies nativas potencialmente úteis, mas também o conhecimento que orienta seu uso, obtido pela experiência de contato estreito com seu ambiente transmitido de geração para geração.

Esta pesquisa foi desenvolvida na comunidade caiçara chamada Praia do Sono, situada na Reserva Ecológica da Juatinga (REJ), no litoral sul do Rio de Janeiro. O levantamento bibliográfico indicou que o conhecimento acumulado sobre as relações entre as populações caiçaras e o meio natural em que estão inseridas se restringe às áreas situadas dentro de Unidades de Conservação, e evidenciou a ausência de estudos sobre estas relações na comunidade caiçara da Praia do Sono.

A pesquisa etnobotânica numa comunidade ainda não estudada é uma oportunidade de acessar uma riqueza de informações de cunho etnográfico e de recursos biológicos, que podem servir de subsídios para trabalhos mais focados em determinados aspectos, como, por exemplo, a bioprospecção de novos fármacos ou manejo de áreas de conservação (Diegues \& Viana 2004).

A cultura caiçara surgiu na Mata Atlântica do litoral dos estados de São Paulo, Rio de Janeiro e Paraná, da miscigenação entre índios, colonizadores portugueses e, em menor grau, escravos africanos (Sanches 2004). Segundo Diegues \& Arruda (2001), tal identidade cultural tem adquirido força nos discursos políticos com o objetivo de dar aos caiçaras um peso e importância equivalentes, na forma da lei, às culturas indígena e quilombola.

Como base da sobrevivência da unidade familiar estão as atividades de agricultura itinerante de corte-queima, pesca e extração de recursos do ambiente, como plantas empregadas para fins alimentares e medicinais (Begossi 1998). Segundo Willems (2003), esta dependência dos recursos naturais para a sobrevivência atribui aos caiçaras um caráter altamente adaptativo ao ambiente no qual estão inseridos.

Adams (2000), ao rever a literatura sobre os caiçaras, levantou 180 publicações entre 1934 e 2000. Diegues \& Arruda (2001) realizaram um levantamento bibliográfico indicando 104 trabalhos significativos sobre as comunidades caiçaras e sua cultura, sendo $15 \%$ destes específicos para o litoral do Rio de Janeiro.

O presente trabalho objetiva investigar o conhecimento sobre as plantas medicinais na comunidade caiçara da Praia do Sono. Levando-se em consideração que o grupo estudado interage diretamente com o bioma da Mata Atlântica, acumulando amplo saber sobre suas espécies vegetais, buscou-se especificamente: registrar as espécies medicinais cultivadas e as que são obtidas pelo extrativismo em áreas de vegetação nativa; analisar a importância relativa de cada táxon citado pelos informantes e avaliar a concordância de uso popular para eleger plantas medicinais nativas com potencial para estudos farmacológicos.

\section{Material e métodos}

\section{Área de estudo}

A Praia do Sono localiza-se na Reserva Ecológica da Juatinga (REJ) que está situada na Área de Preservação Ambiental do Cairuçu (APAC), no município de Paraty, Estado do Rio de Janeiro. Esta praia encontra-se localizada nas coordenadas $23^{\circ} 19^{\prime} 55,2^{\circ}$ de latitude sul e $44^{\circ} 37^{\prime} 57,8^{\circ}$ de longitude oeste e possui 1365 metros de extensão.

A APAC compõe-se de uma parte continental de 33.800 ha e de uma parte insular, com 62 ilhas, (Marques et al. 1997). Sobrepõe-se parcialmente ao Parque Nacional da Serra da Bocaina, e abrange totalmente a REJ que tem uma área de 8.000 ha.

A REJ é constituída por numerosas praias que permeiam os costões rochosos cuja ponta mais extrema empresta o nome à mesma, "Ponta da Juatinga", e abriga ainda 12 núcleos de ocupação de populações tradicionais, que se distribuem, em sua maioria, ao longo do litoral, em cinco principais núcleos: Praia do Sono; Ponta Negra; Praia Grande do Pouso da Cajaíba; Ponta da Juatinga e Mamanguá. Os núcleos se relacionam entre si e usam a cidade de Paraty como centro de comércio e serviço, apesar da precariedade de acesso (SEMADS-RJ 2001).

Aspectos históricos e sócio-econômicos - Há cinqüenta anos moravam no local 220 famílias, que viviam da pesca artesanal e agricultura, constituindo uma comunidade caiçara tradicional. Com a abertura da BR 101, um grande especulador comprou as terras e indenizou 203 famílias, ficando apenas 17 delas que lutaram bravamente pela posse de suas propriedades. Hoje, apesar de não haver um levantamento atualizado, estima-se a população da Praia do Sono em 60 famílias e 350 habitantes.

A comunidade dispõe de uma escola que atende crianças do ensino fundamental, depois disso os alunos têm que recorrer a outros locais e, conseqüentemente, a maioria abandona os estudos. $\mathrm{O}$ atendimento de saúde é praticamente nulo, pois não há posto de saúde. Geralmente os moradores utilizam os conhecimentos tradicionais para se tratar e se for algo mais sério, vão ao hospital de Paraty. Na Praia do Sono não existe sistema de captação de água, tratamento de esgoto ou energia elétrica e toda a infra-estrutura é muito precária.

A organização social do grupo é baseada na unidade familiar e, de modo geral, está de acordo com as descrições propostas por Sanches (2004), que definem as comunidades 
caiçaras presentes na Mata Atlântica. Os laços de solidariedade são muito presentes e podem ser observados nas constantes práticas de: (a) mutirão para a construção ou ampliação das moradias; (b) puxada coletiva de canoas; (c) coleta de barro, (d) de madeiras como jacatirão (Miconia cinnamomifolia Triana), chile ou casca-preta (Pera glabrata (Schott) Baill.), simbiíba ou simbiúva (Hirtella hebeclada Moric. ex DC.), dentre outras e (e) do sapê (Imperata brasiliensis Trin.), nos meses de setembro a dezembro, na época da seca.

Diversos depoimentos relatam que o cultivo de roça diminuiu drasticamente, mas ainda é possível encontrar em quintais alguns pequenos plantios de cana (Saccharum officinarum L.), feijão (Phaseolus vulgaris L.) e milho (Zea mays L.), geralmente para consumo próprio, e de mandioca (Manihot esculenta Crantz). A produção de farinha de mandioca é feita na única casa de farinha existente na comunidade, que recorda a tradição da cultura alimentar. Os moradores da Praia do Sono associam a diminuição do número de roças a duas questões: as restrições ambientais e a diminuição da mão-de-obra, visto que as novas gerações não se interessam mais pela lavoura, dando preferência às atividades remuneradas.

O turismo é uma atividade que vem crescendo nos últimos anos, com um significado econômico muito importante para a comunidade, que aposta nele como via para a conquista de uma vida melhor. Esta atividade se configura na forma de aluguel de chalés, casas e quintais para camping, na oferta de refeições e no serviço de transporte e passeios de bote.

A dificuldade de acesso, a ausência de energia elétrica e as tarefas dos moradores envolvendo o ambiente, são agentes facilitadores que contribuem para que a comunidade da Praia do Sono sustente hábitos e tradições de antigas gerações. Porém, os avanços da tecnologia vêm propiciando o acesso ao local, com a substituição das canoas a remo pelos botes a motor, o que acelera o translado dos habitantes e dos turistas. Pode-se dizer que atualmente os caiçaras da Praia do Sono encontram-se à margem da sociedade global, tanto espacial como socialmente, refletindo um momento dinâmico de transformação e resistência sócio-cultural, seja no modo como expressam seus valores e costumes seja como se apropriam de outros.

\section{Coleta dos dados}

O trabalho de campo compreendeu o período de fevereiro de 2008 a junho de 2009. As visitas à comunidade foram mensais e costumavam durar em média quatro dias. Visitas prévias foram realizadas a fim de conhecer melhor a comunidade e seus códigos de conduta.

Para alcançar a lista de informantes especialistas em potencial, foram utilizadas as técnicas de bola de neve (Bernard 1995) e busca ativa (Albuquerque et al. 2008), com o intuito de priorizar as pessoas que ainda têm o hábito de utilizar plantas no seu dia-a-dia. Os dados foram obtidos através das técnicas de observação direta, participante (Cotton 1996) e registro de entrevistas por meio de um gravador, com o consentimento prévio dos informantes. As entrevistas foram conduzidas com base de um questionário semi-estruturado (Albuquerque et al. 2008; Martin 1995).

Com intuito de coletar o material botânico indicado pelos informantes foram conduzidas incursões à mata utilizando-se a técnica denominada "walking in the woods" de Alexiades \& Sheldon (1996). As espécies da praia e as cultivadas nos quintais dos entrevistados e as informações a respeito destas também foram coletadas. As visitas à mata foram acompanhadas por cinco dos seis homens que participaram da pesquisa e realizadas nos trechos da floresta mais freqüentados por eles. Já as áreas de praia e quintais foram percorridas com todos os 12 informantes.

Todo o material botânico coletado foi herborizado, identificado e depositado no herbário do Museu Nacional/ UFRJ (R). A identificação botânica foi feita a partir de bibliografia específica para cada grupo, da comparação com exemplares dos acervos dos herbários do Museu Nacional (R) e do Jardim Botânico do Rio de Janeiro (RB) e da consulta a especialistas. $\mathrm{O}$ sistema de classificação adotado segue o APG III (2009). A atualização nomenclatural foi conferida através das bases de dados W3 tropicos (Missouri Botanical Garden VAST - VAScular trópicos) e IPNI (The International Plants Names Index).

\section{Análise dos dados}

Buscou-se classificar o "status" das plantas coletadas, sendo consideradas as seguintes definições: plantas nativas, aquelas pertencentes ao domínio da Mata Atlântica e plantas exóticas, as espécies oriundas de outras áreas, segundo as obras de Corrêa (1926-1978) e Stehmann et al. (2009).

As indicações terapêuticas das plantas medicinais indicadas foram decodificadas e agrupadas de acordo com o sistema biomédico convencional, seguindo a "Classificação Internacional de Doenças e Problemas Relacionados à Saúde" (CID) adotada pela Organização Mundial de Saúde (OMS) (WHO 2007), sempre buscando respeitar as informações dos entrevistados e minimizar possíveis distorções.

Algumas vezes os sintomas eram pouco específicos, o que dificultava a categorização dos sistemas corporais. Portanto, quando necessário, uma pesquisa mais profunda era feita com os informantes através de perguntas que possibilitavam esclarecer minimamente a questão.

A fim de avaliar a importância relativa de cada espécie medicinal citada pelos informantes, foi calculado o Índice de Importância Relativa (IR) de Bennett \& Prance (2000), onde o valor máximo alcançado por uma espécie corresponde a 2,00 e é obtido através da fórmula:

$I R=N S C+N P$,

Onde, NSC é igual ao número de sistemas corporais tratados por uma espécie (NSCE) dividido pelo número 
total de sistemas corporais tratados pela espécie mais versátil (NSCEV); e NP é igual ao número de sintomas atribuídos a uma espécie (NPE) dividido pelo número total de sintomas atribuídos à espécie mais versátil (NPEV).

Para analisar a concordância entre as respostas dos informantes sobre o uso medicinal das plantas, o fator CUPc foi calculado para as plantas medicinais citadas por três ou mais informantes através da fórmula (Amorozo \& Gély 1988):

CUP $=$ Fid $/ F e X 100$

Onde, Fid é o número de informantes que citaram usos principais, e $\mathrm{Fe}$ é o número de informantes que citaram o uso da espécie.

Para evitar distorções entre plantas citadas por muitos informantes e as citadas por poucos informantes, o valor de CUP encontrado foi multiplicado por um fator de correção $(F C)$, que corresponde a razão do número de informantes que citaram uma dada espécie pelo número de informantes que citaram a espécie mais citada. Assim, encontra-se o valor de "CUP corrigido" (CUPc):

CUPc $=$ CUP X FC

\section{Resultados e discussão}

Foram entrevistados 12 informantes $(n=12)$ que detinham o conhecimento da vegetação local, com idade entre 29 e 88 anos, sendo 6 homens e 6 mulheres. Dez deles são nativos da Praia do Sono, e apenas dois vieram de regiões circunvizinhas, sendo que residem na comunidade com tempo superior a 50 anos.

Os entrevistados relataram que aprenderam o uso das plantas com os pais e avós que as utilizavam no cotidiano, como pode ser observado nas seguintes falas: "Tudo que aprendi de planta foi com minha mãe e minha avó, eu prestava atenção no que elas usavam e acabei aprendendo também" (Sra. E.); "Meu pai era do tempo dos antigos, tudo era uma dificuldade aqui e eles usava as pranta pra tudo. Aprendi usar com ele" (Sr. D.). Entretanto, a observação de campo indica que as informações sobre as plantas também fluem através de redes informais de conhecimentos, onde saberes e práticas são difundidos entre vizinhos, amigos e familiares de forma muito dinâmica, por meio de trocas de mudas, sementes e receitas.

Foram registradas 89 espécies medicinais distribuídas em 43 famílias botânicas (Tab. 1). As famílias mais representativas em número de espécies foram Lamiaceae (11 spp.) e Asteraceae (7 spp.). O uso de plantas medicinais das famílias Lamiaceae e Asteraceae é predominante em vários levantamentos etnobotânicos realizados em diferentes regiões do país (Moerman et al. 1999; Begossi et al. 2002; Di Stasi et al. 2002; Gazzaneo et al. 2005; Pasa et al. 2005; Boscolo \& Senna-Valle 2008).

Considerando a origem das espécies, os informantes do presente estudo citaram 50 espécies exóticas e 39 nativas. Begossi et al. (2002) estudaram diversas comunidades caiçaras ao longo da costa sudeste do país e identificaram um total de 227 espécies indicadas como de uso medicinal, sendo 38 destas nativas da Mata Atlântica; Silva \& Proença (2008) ao analisarem o uso e disponibilidade de recursos medicinais no município de Ouro Verde de Goiás apuraram 77 plantas exóticas e 21 nativas.

Em relação ao hábito, $51 \%$ das espécies são herbáceas. As arbóreas representam 29\% do total e as arbustivas e trepadeiras $10 \%$ cada. As espécies herbáceas investem em compostos secundários de alta atividade biológica, como alcalóides, glicosídeos e terpenóides, ao invés de desenvolver sistemas de defesas estruturais e de alto peso molecular, como taninos e ligninas, o que explica a grande utilização das ervas nos sistemas médicos populares (Stepp \& Moerman 2001).

Nas indicações dos informantes encontram-se 10 diferentes partes e exudados vegetais utilizados medicinalmente. A folha foi a mais citada (59\%), seguida por planta toda (14\%) e caule (9\%). Trabalhos etnobotânicos na Mata Atlântica freqüentemente encontram a folha como a parte do vegetal mais utilizada nas preparações dos remédios caseiros (Costa-Neto \& Oliveira 2000; Di Stasi \& Lima 2002). Este resultado pode estar relacionado a maior facilidade na obtenção de folhas em relação as demais partes do vegetal, principalmente flores e frutos.

Os dados mostram que $85 \%$ das espécies com propriedade medicinal são cultivadas nos quintais dos informantes e destinadas para consumo do núcleo familiar ou da vizinhança. Destas, $64 \%$ são exóticas e $36 \%$ nativas. O cultivo nos quintais é, notadamente, resultante da comodidade que ele representa, em razão dos variados impedimentos de deslocamento dos especialistas locais, tanto para as áreas mais distanciadas de mata, onde poderiam extrair espécies nativas para suprir demandas eventuais, como para Paraty, onde poderiam adquirir as plantas no comércio da cidade.

O extrativismo dos recursos medicinais não foi observado como uma prática comum, restringindo-se apenas a coletas ocasionais, decorrentes de uma necessidade específica. Christo et al. (2006) consideram que as comunidades situadas em áreas de domínio de Mata Atlântica, cuja diversidade de táxons e potencialidades de uso são elevadas mas que não possuem o costume de retirar plantas da floresta, pode tornar-se um fato preocupante a medida em que a flora nativa perde importância em detrimento do uso de espécies cultivadas, contribuindo para a perda do conhecimento tradicional sobre a vegetação local. Diversos autores concordam que a degradação do meio ambiente, acarretando a redução da disponibilidade de plantas nativas para fins medicinais, juntamente com a facilidade de acesso aos meios de comunicação e o contato com sistemas culturais distintos, são importantes fatores responsáveis para a deterioração do saber tradicional sobre os recursos vegetais (Amorozo 2002; Laraia 2002; Christo et al. 2006).

As indicações terapêuticas tratadas com as 89 plantas medicinais citadas neste trabalho foram agrupadas em 13 categorias de doenças segundo a CID. As categorias mais citadas foram: a que trata do sistema gastrintestinal, com 89 citações, seguida da relacionada às doenças da pele e 
Tabela 1. Plantas medicinais citadas pelos informantes da Praia do Sono, Paraty, RJ. Família / espécie. Nome local. "Status". Parte utilizada. Indicação. Local de coleta.

\begin{tabular}{|c|c|c|c|c|c|}
\hline Família / espécie & Nome local & "Status" & Parte utilizada & Indicação & Local de coleta \\
\hline \multicolumn{6}{|l|}{ ALISMATACEAE } \\
\hline Echinodorus grandiflorus Mitch. & chapéu-de-couro & nativa & folha & fortificante; problemas nos rins & quintal \\
\hline \multicolumn{6}{|l|}{ AMARANTHACEAE } \\
\hline Alternanthera brasiliana (L.) O. Kuntze & terramicina & nativa & folha & $\begin{array}{l}\text { antiinflamatório; infecção urinária; } \\
\text { inflamação do útero; infecção de garganta; } \\
\text { machucadura; cicatrizante }\end{array}$ & quintal \\
\hline Iresine herbstii Hook. & arnica; paraguaia & exótica & folha & machucadura & quintal \\
\hline \multicolumn{6}{|l|}{ AMARYLLIDACEAE } \\
\hline Allium sativum $\mathrm{L}$. & alho & exótica & caule & dor de garganta & quintal \\
\hline \multicolumn{6}{|l|}{ ANACARDIACEAE } \\
\hline Anacardium occidentale $\mathrm{L}$. & caju & nativa & fruto & ferimentos na boca & quintal \\
\hline Mangifera indica $\mathrm{L}$. & manga & exótica & folha & dente inflamado & quintal \\
\hline Schinus terebinthifolius Raddi & aroeira & nativa & caule & inflamação no útero; problema no útero & praia \\
\hline \multicolumn{6}{|l|}{ ANNONACEAE } \\
\hline Guatteria australis A.St.-Hil. & bicuíba & nativa & fruto & ferimento & mata \\
\hline \multicolumn{6}{|l|}{ ASTERACEAE } \\
\hline Achyrocline satureoides (Lam.) DC. & camomila; macela & nativa & inflorescência & $\begin{array}{c}\text { calmante; baixar a pressão; problema } \\
\text { nos rins }\end{array}$ & mata \\
\hline Ageratum conyzoides $\mathrm{L}$. & erva-de-são-joão & nativa & planta toda & cicatrizante; dor de estômago & quintal \\
\hline Baccharis trimera (Less.) DC. & carqueja & exótica & caule & doenças do fígado & quintal \\
\hline Bidens pilosa $\mathrm{L}$. & picão & nativa & planta toda & $\begin{array}{l}\text { doenças do fígado; dor de urina; anemia; } \\
\text { hepatite; ictericia }\end{array}$ & quintal \\
\hline Mikania glomerata Spreng. & guaco & nativa & folha & gripe & quintal \\
\hline Vernonia beyrichii Less. & cambará-roxo & nativa & folha & catarro no peito & mata \\
\hline Vernonia polyanthes Less. & assa-peixe & exótica & folha & gripe & quintal \\
\hline \multicolumn{6}{|l|}{ BIGNONIACEAE } \\
\hline Crescentia cujete $\mathrm{L}$. & cabaço & exótica & fruto & expulsar a placenta após o parto; anemia & quintal \\
\hline \multicolumn{6}{|l|}{ BORAGINACEAE } \\
\hline Cordia verbenacea DC. & baleeira & exótica & folha & contusão & quintal \\
\hline \multicolumn{6}{|l|}{ BRASSICACEAE } \\
\hline Brassica oleracea L. var. acephala D.C. & couve & exótica & folha & anemia & quintal \\
\hline Nasturtium officinale R. Br. & agrião & exótica & folha & bronquite & quintal \\
\hline \multicolumn{6}{|l|}{ BROMELIACEAE } \\
\hline Ananas comosus (L.) Merr. & abacaxi & exótica & fruto & pneumonia & quintal \\
\hline \multicolumn{6}{|l|}{ CARICACEAE } \\
\hline Carica papaya L. & mamão-macho & exótica & flor & tosse; gripe; coqueluche & quintal \\
\hline \multicolumn{6}{|l|}{ CECROPIACEAE } \\
\hline Cecropia pachystachya Trec. & baibera; imbaíba & nativa & folha & tosse & mata; quintal \\
\hline \multicolumn{6}{|l|}{ CHENOPODIACEAE } \\
\hline Chenopodium ambrosioides $\mathrm{L}$. & santa-maria & exótica & parte aérea & machucadura & quintal \\
\hline \multicolumn{6}{|l|}{ CONVOVULACEAE } \\
\hline Ipomea batatas (L.) Lam. & batata-doce & exótica & raíz & dente inflamado & quintal \\
\hline \multicolumn{6}{|l|}{ COSTACEAE } \\
\hline Costus spicatus (Jacq.) Sw. & cana-do-brejo & nativa & folha & problema nos rins & quintal \\
\hline \multicolumn{6}{|l|}{ CRASSULACEAE } \\
\hline Bryophyllum pinnatum (L. f.) Oken & estalo & exótica & folha & frieira & mata; quintal \\
\hline
\end{tabular}


Tabela 1. Continuação

\begin{tabular}{|c|c|c|c|c|c|}
\hline Família / espécie & Nome local & "Status" & Parte utilizada & Indicação & Local de coleta \\
\hline Kalanchoe brasiliensis Cambess & saião & nativa & folha & expectorante; tosse; machucadura; frieira & quintal \\
\hline \multicolumn{6}{|l|}{ CUCURBITACEAE } \\
\hline Cucurbita pepo L. & abóbora & exótica & flor & dor de ouvido & quintal \\
\hline Momordica charantia $\mathrm{L}$. & $\begin{array}{l}\text { melão-de-são- } \\
\text { caetano }\end{array}$ & exótica & folha & reumatismo hemorroida & quintal \\
\hline Sechium edule (Jacq.) Sw. & chuchu & exótica & folha & pressão alta; cólica & quintal \\
\hline \multicolumn{6}{|l|}{ DILLENIACEAE } \\
\hline Davilla rugosa Poiret & cipó-caboclo & nativa & caule & bom pra vista & mata \\
\hline \multicolumn{6}{|l|}{ ERYTROXYLACEAE } \\
\hline Erytroxylum ovalifolium Peyr. & $\begin{array}{l}\text { futiabeira; } \\
\text { putiabeira }\end{array}$ & nativa & folha & $\begin{array}{c}\text { machucadura; cicatrizante de ferida; } \\
\text { antiinflamatório }\end{array}$ & quintal \\
\hline \multicolumn{6}{|l|}{ EUPHORBIACEAE } \\
\hline Euphorbia prostrata Aiton & quebra-pedra & nativa & planta toda & $\begin{array}{c}\text { dor de urina; problemas nos rins; infecção } \\
\text { na urina }\end{array}$ & quintal \\
\hline Ricinus communis $\mathrm{L}$. & mamona & exótica & folha & dor de cabeça & quintal \\
\hline \multicolumn{6}{|l|}{ FABACEAE } \\
\hline Cajanus cajan (L.) Millsp. & feijão-guandu & exótica & folha & dor de dente; pressão & quintal \\
\hline Desmodium adscendens (SW.) DC. & carrapichinho & nativa & planta toda & dor de urina & mata \\
\hline Hymenaea courbaril L. & jatobá & nativa & caule & fortificante & mata \\
\hline Mimosa pudica L. & dormideira & nativa & planta toda & $\begin{array}{c}\text { inflamação no dente; inflamação na } \\
\text { garganta }\end{array}$ & quintal \\
\hline \multicolumn{6}{|l|}{ LAMIACEAE } \\
\hline Leonotis nepetaefolia (L.) R. Br. & cordão-de-frade & exótica & folha & inflamação na urina & quintal \\
\hline Leonurus sibiricus L. & santa-rita & exótica & planta toda & dor de estômago & quintal \\
\hline $\begin{array}{l}\text { Marsypianthes chamaedrys (Vahl.) } \\
\text { Kuntze }\end{array}$ & erva-madre & nativa & planta toda & corrimento & quintal \\
\hline Mentha pulegium $\mathrm{L}$. & poejo & exótica & planta toda & tosse & quintal \\
\hline Mentha sativa $\mathrm{L}$. & $\begin{array}{l}\text { hortelã-de-bicho; } \\
\text { hortelã-miúdo; } \\
\text { hortelãzinho; } \\
\text { hortelã }\end{array}$ & exótica & folha & tosse; verme & quintal \\
\hline Ocimum basilicum $\mathrm{L}$. & manjericão & exótica & folha & dor de dente; má digestão & quintal \\
\hline Ocimum selloi Benth. & alfavaca & nativa & folha & tosse; gripe & quintal \\
\hline Plectranthus amboinicus (Lour.) Spreng. & hortelã-de-galinha & exótica & folha & tosse; dor de barriga & quintal \\
\hline Plectranthus barbatus Andrews & boldo & exótica & folha & $\begin{array}{c}\text { cólica; dor no estômago; ressaca; doenças } \\
\text { do fígado }\end{array}$ & quintal \\
\hline Plectranthus ornatus Codd. & anador & exótica & folha & $\begin{array}{c}\text { dor de estômago; dor no fígado; dor de } \\
\text { dente; dor de cabeça }\end{array}$ & quintal \\
\hline Rosmarinus officinalis $\mathrm{L}$. & alecrim & exótica & folha & má digestão & quintal \\
\hline \multicolumn{6}{|l|}{ LAURACEAE } \\
\hline Laurus nobilis $\mathrm{L}$. & louro & exótica & folha & gases & quintal \\
\hline Persea americana Mill. & abacate & exótica & folha & $\begin{array}{c}\text { problema nos rins; soltar a urina; infecção } \\
\text { urinária; doenças do fígado; tosse ; } \\
\text { reumatismo }\end{array}$ & quintal \\
\hline \multicolumn{6}{|l|}{ LECYTHIDACEAE } \\
\hline Cariniana estrellensis (Raddi) Kuntze & jequitibá & nativa & caule & infecção no útero & mata \\
\hline \multicolumn{6}{|l|}{ LORANTHACEAE } \\
\hline Struthanthus concinnus Mart. & $\begin{array}{l}\text { erva-de- } \\
\text { passarinho }\end{array}$ & nativa & folha & machucadura; dor no peito, dor na costela & quintal \\
\hline
\end{tabular}


Tabela 1. Continuação

\begin{tabular}{|c|c|c|c|c|c|}
\hline Família / espécie & Nome local & "Status" & Parte utilizada & Indicação & Local de coleta \\
\hline \multicolumn{6}{|l|}{ LYTRACEAE } \\
\hline Punica granatum $\mathrm{L}$. & romã & exótica & fruto & garganta inflamada & quintal \\
\hline \multicolumn{6}{|l|}{ MALVACEAE } \\
\hline Gossypium herbaceum L. & algodão & exótica & folha & $\begin{array}{l}\text { infecção no útero; inflamação no útero; } \\
\text { cicatrizante }\end{array}$ & mata \\
\hline Sida planicaulis Cav. & vassoura & nativa & folha & puxar o puz do furúnculo & quintal \\
\hline \multicolumn{6}{|l|}{ MORACEAE } \\
\hline Artocarpus heterophyllus Lam. & jaca & exótica & folha & erisipela & quintal \\
\hline Morus nigra L. & amora & exótica & folha & $\begin{array}{l}\text { aliviar as coisas da menopausa; doenças } \\
\text { dos rins }\end{array}$ & quintal \\
\hline Sorocea guilleminiana Gaudich & espinheira-santa & nativa & folha & problemas no estômago; úlcera & mata \\
\hline \multicolumn{6}{|l|}{ MUSACEAE } \\
\hline Musa X paradisiaca L. & banana & exótica & folha & estancar o sangue & quintal \\
\hline \multicolumn{6}{|l|}{ MYRTACEAE } \\
\hline Eucalyptus sp. & eucalipto & exótica & folha & rinite; sinusite - inalação & quintal \\
\hline Eugenia uniflora L. & pitanga & nativa & folha & tosse; expectorante; febre; diarréia & quintal \\
\hline Plinia edulis (O. Berg) Nied & cambucá & nativa & folha & tosse & quintal \\
\hline Psidium guajava $\mathrm{L}$. & goiaba & exótica & folha & diarréia; dor de barriga & quintal \\
\hline \multicolumn{6}{|l|}{ PASSIFLORACEAE } \\
\hline Passiflora alata Curtis & maracujá & nativa & folha & calmante & quintal \\
\hline Passiflora edulis Sims & maracujá & nativa & folha & calmante & quintal \\
\hline \multicolumn{6}{|l|}{ PHYLLANTHACEAE } \\
\hline Phyllanthus tenellus Roxb. & quebra-pedra & nativa & planta toda & $\begin{array}{c}\text { dor de urina; problemas nos rins; infecção } \\
\text { na urina }\end{array}$ & quintal \\
\hline \multicolumn{6}{|l|}{ PIPERACEAE } \\
\hline Piper mollicomum Kunth & joão-borandí & nativa & folha & queda de cabelo & quintal \\
\hline Pothomorphe umbellata (L.) Miq. & pariparoba & nativa & raíz & anemia & mata \\
\hline \multicolumn{6}{|l|}{ POACEAE } \\
\hline Cymbopogum citratus (DC.) Stapf. & $\begin{array}{l}\text { capim-cidreira; } \\
\text { capim-cidão }\end{array}$ & exótica & folha & $\begin{array}{l}\text { diarréia; dor de estômago; cólica } \\
\text { intestinal; vômito; calmante }\end{array}$ & quintal \\
\hline Eleusine indica (L.) Gaertn. & pé-de-galinha & exótica & planta toda & esporão; osteoporose & quintal \\
\hline \multicolumn{6}{|l|}{ POLYGALACEAE } \\
\hline Polygala paniculata $\mathrm{L}$. & arnica; gelol & nativa & raíz & contusão & quintal \\
\hline \multicolumn{6}{|l|}{ ROSACEAE } \\
\hline Rosa canina $\mathrm{L}$. & rosa-branca & exótica & flor & $\begin{array}{l}\text { infecção de urina; bom pra vista; dente } \\
\text { inflamado; inflamação na garganta; ferida } \\
\text { na boca }\end{array}$ & quintal \\
\hline Rubus rosifolius $\mathrm{Sm}$. & amora-de-espinho & nativa & planta toda & $\begin{array}{c}\text { dente inflamado; ferida na boca; infecção } \\
\text { de urina }\end{array}$ & mata \\
\hline \multicolumn{6}{|l|}{ RUBIACEAE } \\
\hline Coffea arabica $\mathrm{L}$. & café & exótica & semente & problema na vista & quintal \\
\hline \multicolumn{6}{|l|}{ RUTACEAE } \\
\hline Citrus aurantium $\mathrm{L}$. & $\begin{array}{l}\text { laranja-china; } \\
\text { laranja-lima }\end{array}$ & exótica & folha & gripe; gripe com febre & quintal \\
\hline Citrus deliciosa Ten. & $\begin{array}{l}\text { laranja-crava; } \\
\text { mixirica }\end{array}$ & exótica & folha & gripe & quintal \\
\hline Citrus limon (L.) Burm. f. & limão & exótica & folha & $\begin{array}{l}\text { resfriado; aumentar o apetite; tirar acidez } \\
\text { da boca; inflamação na garganta }\end{array}$ & quintal \\
\hline
\end{tabular}


Tabela 1. Continuação

\begin{tabular}{|c|c|c|c|c|c|}
\hline Família / espécie & Nome local & "Status" & Parte utilizada & Indicação & Local de coleta \\
\hline Citrus reticulata Blanco & pocã & exótica & folha & gripe & quintal \\
\hline Citrus sinensis (L.) Osbeck & $\begin{array}{l}\text { laranja-baía; } \\
\text { laranja }\end{array}$ & exótica & folha & gripe & quintal \\
\hline Ruta graveolens L. & arruda & exótica & folha & febre & quintal \\
\hline \multicolumn{6}{|l|}{ SOLANACEAE } \\
\hline Solanum americanum Mill. & maria-preta & nativa & folha & machucadura & quintal \\
\hline Solanum sisymbrifolium Lam. & rebenta-cavalo & nativa & fruto & $\begin{array}{l}\text { coloca no furúnculo para puxar o puz; } \\
\text { inflamação no dente }\end{array}$ & quintal \\
\hline \multicolumn{6}{|l|}{ ULMACEAE } \\
\hline Trema micrantha (L.) Blume & candiúba & nativa & caule & icterícia; hemorróida & mata \\
\hline \multicolumn{6}{|l|}{ VERBENACEAE } \\
\hline Lippia alba (Mill.) N. E. Br. & erva-cidreira & nativa & folha & $\begin{array}{c}\text { pressão alta; cólica menstrual; calmante; } \\
\text { dor de estômago; cólica intestinal; vômito; } \\
\text { diarréia }\end{array}$ & quintal \\
\hline $\begin{array}{l}\text { Stachytarpheta cayennensis (Rich.) } \\
\text { Vahl. }\end{array}$ & gervão & nativa & planta toda & anemia & quintal \\
\hline \multicolumn{6}{|l|}{ XANTHORRHOEACEAE } \\
\hline Aloe vera (L.) Burm. f. & babosa & exótica & mucilagem & queimadura; queda de cabelo & quintal \\
\hline \multicolumn{6}{|l|}{ ZINGIBERACEAE } \\
\hline Hedychium coronarium J.Koenig & ciosa & exótica & flor & coqueluche & quintal \\
\hline Zingiber officinale Roscoe & gengibre & exótica & caule & reumatismo; artrite & quintal \\
\hline
\end{tabular}

do tecido subcutâneo, com 60 citações e da que inclui as patologias associadas ao sistema respiratório, com 58 citações. As doenças mais citadas na presente pesquisa também aparecem como as mais comuns no Pará (Amorozo \& Gély, 1988), no Mato Grosso (Amorozo 2002) e na região sudeste do país (Medeiros et al. 2004; Pilla et al. 2006).

Bennet \& Prance (2000) assumem que a planta mais importante numa comunidade é aquela que for mais versátil, ou seja, a planta que for utilizada para tratar a maior variedade de doenças, sendo, portanto, considerada um "remédio milagroso". No entanto, se o objetivo for a busca de novas drogas, a concordância das respostas dos informantes sobre o uso medicinal de uma determinada planta é extremamente importante, já que plantas utilizadas para muitos fins teriam uma menor credibilidade quando comparadas aquelas com maior fidelidade de uso.

Lippia alba (Mill.) N. E. Br.(erva-cidreira) apresentou o maior valor de Importância Relativa ( $I R=2,0$ ), ou seja, foi a espécie indicada para o tratamento de um maior número de sintomas, seguida de Alternanthera brasiliana (L.) O. Kuntze (terramicina) e Persea americana Mill. (abacate), ambos com IR = 1,57 (Tab. 2).

Dez espécies apresentaram grande versatilidade quanto aos seus usos, com IR $>1$, sendo indicadas para até 10 sistemas corporais. $\mathrm{O}$ uso de plantas com IR $>1$ também vem sendo relatado em outras regiões do país, como exemplifica o estudo de Almeida \& Albuquerque (2002) realizado na feira de Caruaru, estado de Pernambuco, que ap resentou nove espécies com IR > 1 para o tratamento de até oito categorias de doença.
O fator de Concordância de Uso Principal corrigido (CUPc) aponta os usos mais difundidos e aceitos para uma espécie, já que são utilizados e reconhecidos pelos informantes, o que poderia evidenciar maior segurança quanto à sua validade (Vendruscolo \& Mentz 2006). Assim, este fator tem sido utilizado para indicar quais plantas devem ter prioridade de estudos, ou seja, direcionar os estudos para bioprospecção (Thring \& Weitz 2006).

As espécies com CUPc maior que 50\% apresentam um apreciável consenso de uso popular, o que pode indicar potencial medicinal, funcionando como uma pré-triagem de espécies para estudos etnofarmacológicos (Silva \& Proença 2008). Nesse estudo, 13 espécies apresentaram um alto consenso de informações, o que pode implicar maior eficácia quanto ao uso (Tab. 3).

Duas espécies medicinais oriundas exclusivamente da mata, e, portanto, obtidas por extrativismo, apresentaram a Concordância de Uso Principal corrigido com valores acima de 50\%: Davilla rugosa Poiret e Trema micrantha (L.) Blume (ambas com CUPc $=55,6 \%$ ). Cecropia pachystachya Trec. (também com CUPc $=55,6 \%)$ é coletada tanto na mata quando em quintais.

A partir dos resultados obtidos neste trabalho foi possível concluir que, apesar das diversas influências oriundas da convivência com habitantes de grandes centros urbanos que contribuem com a transformação das tradições locais, as características associadas ao povo caiçara ainda persistem de forma bem evidente na população da Praia do Sono. 
Tabela 2. Índice de Importância Relativa (IR) das espécies medicinais indicadas pelos informantes da Praia do Sono.

\begin{tabular}{|c|c|}
\hline IR & Espécies \\
\hline 2,00 & Lippia alba \\
\hline 1,57 & Alternanthera brasiliana; Persea americana \\
\hline 1,46 & Rosa canina \\
\hline 1,32 & Bidens pilosa; Eugenia uniflora \\
\hline 1,18 & Achyrocline satureoides \\
\hline 1,07 & Citrus limon; Cymbopogum citratus; Plectranthus ornatus \\
\hline 0,93 & Kalanchoe brasiliensis; Rubus rosifolius \\
\hline 0,79 & $\begin{array}{l}\text { Ageratum conyzoides; Cajanus cajan; Carica papaya; Chenopodium ambrosioides; Crescentia cujete; Echinodorus grandiflorus; Erytroxylum } \\
\text { ovalifolium; Mentha sativa; Momordica charantia;Plectranthus amboinicus;Sechium edule; Solanum sisymbrifolium; Struthanthus concinnus }\end{array}$ \\
\hline 0,68 & Plectranthus barbatus \\
\hline 0,54 & $\begin{array}{l}\text { Aloe vera; Eleusine indica; Eucalyptus sp.; Gossypium herbaceum; Mimosa pudica; Morus nigra; Ocimum basilicum; Ocimum selloi; Trema micrantha; } \\
\text { Zingiber officinale }\end{array}$ \\
\hline 0,39 & $\begin{array}{l}\text { Allium sativum; Anacardium occidentale; Ananas comosus; Artocarpus heterophyllus; Baccharis trimera; Brassica oleracea; Bryophyllum pinnatum; } \\
\text { Cariniana estrellensis; Cecropia pachystachya; Citrus aurantium; Citrus deliciosa; Citrus reticulata; Citrus sinensis; Coffea arabica; Cordia verbenacea; } \\
\text { Costus spicatus; Cucurbita pepo; Davilla rugosa; Desmodium adscendens; Euphorbia prostrata; Guatteria australis; Hedychium coronarium; } \\
\text { Hymenaea courbaril; Ipomea batatas; Iresine herbstii; Laurus nobilis; Leonotis nepetaefolia; Leonurus sibiricus; Mangifera indica; Marsypianthes } \\
\text { chamaedrys; Mentha pulegium; Mikania glomerata; Musa X paradisiaca; Nasturtium officinale; Passiflora alata; Passiflora edulis; Phyllanthus } \\
\text { tenellus; Piper mollicomum; Piper umbellata; Plinia edulis; Polygala paniculata; Psidium guajava; Punica granatum; Ricinus communis; Rosmarinus } \\
\text { officinalis; Ruta graveolens; Schinus terebinthifolius; Sida planicaulis; Solanum americanum; Sorocea guilleminiana; Stachytarpheta cayennensis; } \\
\text { Vernonia beyrichii; Vernonia polyanthes }\end{array}$ \\
\hline
\end{tabular}

Tabela 3. Espécies medicinais ordenadas quanto à Concordância de Uso Principal corrigido (CUPc) maior que 50\% entre os informantes da Praia do Sono.

\begin{tabular}{llc}
\hline Espécie & Usos principais & CUPc (\%) \\
\hline Alternanthera brasiliana & machucadura & 88,9 \\
Chenopodium ambrosioides & machucadura; verme & 77,8 \\
Eugenia uniflora & febre & 77,8 \\
Psidium guajava & dor de barriga; diarréia & 77,8 \\
Solanum americanum & machucadura & 77,8 \\
Mentha sativa & verme & 66,7 \\
Carica papaya & tosse & 55,6 \\
Cecropia pachystachya & tosse & 55,6 \\
Davilla rugosa & bom pra vista & 55,6 \\
Erytroxylum ovalifolium & machucadura & 55,6 \\
Ricinus communis & dor de cabeça & 55,6 \\
Sechium edule & pressão alta & 55,6 \\
Trema micrantha & ictericia & 55,6 \\
\hline
\end{tabular}

Os informantes deste estudo indicaram que o uso dos recursos vegetais é uma importante prática desenvolvida por eles e que a transmissão do conhecimento sobre as plantas medicinais se caracteriza por uma mistura de práticas e trocas de saberes entre pais, avós, familiares, amigos e vizinhos, de forma muito dinâmica e informal.

Os elevados valores de CUPc encontrados para os táxons nativos Alternanthera brasiliana (L.) O. Kuntze; Cecropia pachystachya Trec.; Eugenia uniflora L.; Davilla rugosa Poiret; Erytroxylum ovalifolium Peyr. e Trema micrantha (L.) Blume evidenciam que estes merecem atenção especial em estudos etnofarmacológicos, contribuindo para que o conhecimento popular respaldado pelo conhecimento científico colabore para o uso racional de plantas medicinais e para a conscientização da importância de conservá-las.

\section{Agradecimentos}

À comunidade caiçara da Praia do Sono, por compartilharem seus conhecimentos; ao Programa UFRJ/CENPES, pelo apoio financeiro; ao Programa de Pós-Graduação em Botânica do Museu Nacional, pelo apoio e atenção; à pesquisadora Ivone Manzali de Sá, pela revisão do texto; aos 
doutores Maria Christina de Mello Amorozo e Fábio Pedro Souza de Ferreira Bandeira e ao amigo Alexandre Christo, pelas idéias; às amigas Maria Carmem Reis, Veronica MaioliAzevedo e Gabriella Almeida, pelas ajudas no campo.

\section{Referências bibliográficas}

Adams, C. 2000. Caiçaras na Mata Atlântica: pesquisa científica versus planejamento e gestão ambiental. São Paulo, Annablume/FAPESP.

Albuquerque, U.P.; Lucena, R.F.P. \& Cunha, L.V.F.C. (Orgs.). 2008. Métodos e técnicas na pesquisa etnobotânica. Recife, ComunigrafEditora/Nupeea.

Alexiades, M.N. \& Sheldon, J.W. (Eds.). 1996. Selected guidelines for ethnobotanical research: a field manual. The New York Botanical Garden Press. New York, Advances in Economic Botany 10: 1-306.

Almeida, C.F.C.B.R. \& Albuquerque, U.P. 2002. Uso e conservação de plantas medicinais no estado de Pernambuco (Nordeste do Brasil): um estudo de caso. Interciência 27: 276-285.

Amorozo, M.C.M. 2002. Uso e diversidade de plantas medicinais em Santo Antônio do Leverger, MT, Brasil. Acta Botanica Brasilica 16: 189-203.

Amorozo, M.C.M. \& Gély, A. 1988. Uso de plantas medicinais pelos caboclos do baixo Amazonas, Barcarena, Pará, Brasil. Boletim do Museu Paraense Emílio Goeldi, Botânica 4: 47-131.

Angiosperm Phylogeny Group - (APG III). 2009. An update of the angiosperm phylogeny group classification for the orders and families of flowering plants. Botanical Journal of the Linnean Society 161: 105-121.

Bennet, B.C. \& Prance, G.T. 2000. Introduced plants in the indigenous pharmacopoeia of northern South America. Economic Botany 54: 90-102.

Bernard, H.R. 1995. Research methods in anthropology: qualitative and quantitative approaches. Walnut Creek, Altamira Press.

Begossi, A. 1998. Knowledge on the use of natural resources contibutions to local management. In: Cotton, C.M. Ethnobotany - principles and applications. Chichester, John Wiley and Sons.

Begossi, A.; Hanazaki, N. \& Silvano, R.A.M. 2002. Ecologia humana, etnoecologia e conservação. In: Amorozo, M.C.M.; Ming, L.C.; Silva, S.M.P. (Orgs.). Método de coleta e análise de dados em etnobiologia, etnoecologia e disciplinas correlatas - I Seminário de Etnobiologia e Etnoecologia do Sudeste. Rio Claro, CNPq/UNESP.

Begossi, A.; Hanazaki, N. \& Tamashiro, J.Y. 2002. Medicinal Plants in the Atlantic Forest (Brazil): Knowledge, Use, and Conservation. Human Ecology 30: 281-299.

Boscolo, O.H. \& Senna-Valle, L. 2008. Plantas de uso medicinal em Quissamã, Rio de Janeiro, Brasil. Iheringia, sér. Bot. 63(2): 263-277.

Christo, A.G., Guedes-Bruni, R.R. \& Fonseca-Kruel, V.S. 2006. Uso de recursos vegetais em comunidades limítrofes à Reserva Biológica de Poço das Antas, Silva Jardim, Rj: estudo de caso na Gleba Aldeia Velha. Rodriguésia 57: 519-542.

Corrêa, M.P. 1926-1978. Dicionário das plantas úteis do Brasil e das exóticas cultivadas. Rio de Janeiro, Imprensa Nacional.

Costa-Neto, E.M. \& Oliveira, M.V.M. 2000. The use of medicinal plants in the country of Tanquinho, State of Bahia, Northeastern Barzil. Revista Brasileira de Plantas Medicinais 2: 1-6.

Cotton, C.M. 1996. Ehtnobotany: principles and applications. Chichester, John Wiley and Sons.

Diegues, A.C. \& Arruda, R.S.V. 2001. Saberes tradicionais e biodiversidade no Brasil. Brasília: Ministério do Meio Ambiente; São Paulo, USP (Biodiversidade, 4).

Diegues, A.C. \& Viana, V.M. (Orgs.). 2004. Comunidades tradicionais e manejo dos recursos naturais da Mata Atlântica. 2 ed. São Paulo, Editora HUCITEC NUPAUB/CEC.

Di Stasi, L.C. \& Lima, C.A. 2002. Plantas medicinais na Amazônia e na Mata Atlântica. 2 ed. São Paulo, UNESP.

Di Stasi, L.C.;Oliveira, G.P., Carvalhaes, M.A., Queiroz-Junior, M., Tien, O.S., Kakinami, S.H. \& Reis, M.S. 2002. Medicinal plants popularly used in the Brazilian Tropical Atlantic Forest. Fitoterapia 73: 69-91.
Elisabetsky, E. \& Souza, G.C. 2004. Etnofarmacologia como ferramenta na busca de substâncias ativas. In: Simões, C.M.O.; Schenkel, E.P.; Gosmann, G.; Mello, J.C.P.; Mentz, L.A. \& Petrovick, P.R. (Orgs.) Farmacognosia: da planta ao medicamento. 5 ed. Florianopolis, UFSC.

Gazzaneo, L.R.S.; Lucena, R.F.P. \& Albuquerque, U.P. 2005. Knowledge and use of medicinal plants by local specialists in a region of Atlantic Forest in the state of Pernambuco (Northeastern Brazil). Journal of Ethnobiology and Ethnomedicine 1: 1-11.

IPNI - (The International Plants Names Index). Disponível em: < http:// www.ipni.org/>. (Acesso em: 01/2010).

Laraia, R.B. 2002. Cultura: um conceito antropológico. 15 ed. Rio de Janeiro, Jorge Zahar Editora.

Manzali de Sá, I. 2006. A interdisciplinaridade na pesquisa de plantas medicinais de uso tradicional. Revista de Ciências Agroveterinárias 5: 7-12.

Marques, M.C.M.; Vaz, A.S.F. \& Marquete, R. (Orgs). 1997. Flórula da APA de Cairuçu, Paraty, RJ: espécies vasculares. Série estudos e contribuições $\mathrm{n}^{\circ} 14$. Rio de Janeiro, Instituto de Pesquisas Jardim Botânico do Rio de Janeiro.

Martin, G.J. 1995. Ethnobotany: a methods manual. London, Chapman And Hall.

Medeiros, M.F.T., Fonseca, V.S. \& Andreata, R.H.P. 2004. Plantas medicinais e seus usos pelos sitiantes da Reserva Rio das Pedras, Mangaratiba, RJ, Brasil. Acta Botanica Brasilica 18: 391-399.

MMA - (Ministério do Meio Ambiente). 2002. Avaliação e identificação de áreas e ações prioritárias para a conservação, utilização sustentável e repartição dos benefícios da biodiversidade nos biomas brasileiros. Brasília, MMA.

Moerman, D.E.; Pemberton, R.W.; Kiefer, D. \& Berlin, B. 1999. A comparative analysis of five medicinal floras. Journal of ethnobiology 19: 49-67.

Pasa, M.C.; Soares, J.N. \& Guarim Neto G. 2005. Estudos etnobotânicos na comunidade de Conceição- Açu (alto de bacia do rio Ariçá Açu, MT, Brasil). Acta Botanica Brasilica 19: 195-207.

Pilla, M.A.C.; Amorozo, M.C.M. \& Furlan, A. 2006. Obtenção e uso das plantas medicinais no distrito de Martim Francisco, Município de Mogi-Mirim, SP, Brasil. Acta Botanica Brasilica 20: 789-802.

Prance, G.T. 1995. Ethnobotany today and in the future. Pp. 60-67. In: Shultes, R.E. \& Reis, S. Von (Eds.). 1995. Ethnobotany: evolution of a discipline. New York, Chapman \& Hall.

Sanches, R.A. 2004. Caiçaras e a Estação Ecológica de Juréia-Itatins: litoral sul de São Paulo. São Paulo, Annablume, FAPESP.

SEMADS-RJ - (Secretaria de Estado de Meio Ambiente e Desenvolvimento Sustentável do Rio de Janeiro). 2001. Atlas das Unidades de Conservação da Natureza do Estado do Rio de Janeiro. Rio de Janeiro, Metalivros.

Silva, C.S.P. \& Proença, C.E.B. 2008. Uso e disponibilidade de recursos medicinais no município de Ouro Verde de Goiás, GO, Brasil. Acta Botanica Brasilica 22: 481-492.

Stehmann, J.R.; Forzza, R. C.; Salino, A.; Sobral, M.; Da Costa, D.P. \& Kamino, L.H.Y. (Orgs.). 2009. Plantas da Floresta Atlântica. Rio de Janeiro, Jardim Botânico do Rio de Janeiro.

Stepp, J.R. \& Moerman, D.E. 2001. The importance of weeds in ethnopharmacology. Journal of Ethnopharmacology 75: 19-23.

Thring, T.S.A. \& Weitz, F.M. 2006. Medicinal plant use in the bredasdorp/ Elim region of the Southern Overberg in the Western Cape Province of South Africa. Journal of Ethnopharmacology 103: 261-275.

Vendruscolo, G.S. \& Mentz, L.A. 2006. Estudo da concordância das citações de uso e importância das espécies e famílias utilizadas como medicinais pela comunidade do bairro Ponta Grossa, Porto Alegre, RS, Brasil. Acta Botanica Brasilica 20: 367-382.

W3 Tropicos (Missouri Botanical Garden VAST - Vascular Trópicos). Disponível em: <Http://www.tropicos.org/> (Acesso em 01/ 2010).

Willems, E. 2003. A ilha de Búzios: uma comunidade caiçara no sul do Brasil. São Paulo, Editora Hucitec.

World Health Organization - (WHO). 2007. Classificação Internacional de Doenças. Disponível em: <Http://www.who.int/classifications/ apps/icd/icd10online> (Acesso em 01/2009). 